\title{
Medplanner: Android Application for Reminding Users about Medication
}

Corina Holom, Delilah Florea

Samuel von Brukenthal National College of Sibiu

Huet Square, No. 5

Sibiu, Romania

holom_corina@yahoo.com,delilah_florea@yahoo.com

ABSTRACT: Nowadays, life without technology is difficult to envisage for most users and considered a step back. Technology plays a major role in the evolution process and most people embrace it as part of their lives keeping up with all the new developments. Two-thirds of adults worldwide, 66.5 percent, will own smartphones next year, according to new forecasts from media measurement company Zenith. A surmise that explains people's dependence of technology is that it makes their lives easier to the point where even the impossible seems feasible. More and more tasks can be fulfilled faster, safer and more efficiently by computers rather than humans. Technology also makes up for human faults such as forgetfulness, haste and neglect. A mundane task, like taking your medication, can be easily overlooked during a stressful or agitated period. The consequences of this can only be unpleasant and even cause serious problems. Most incommodities can be easily avoided thanks to various newly developed applications and software.

Keywords: Mobile Apps, Graphic Users Interface, Software Design, Reminders

Received: 8 June 2018, Revised 29 July 2018, Accepted 9 August 2018

DOI: $10.6025 / \mathrm{jet} / 2018 / 9 / 4 / 133-138$

(C) 2018 DLINE. All Rights Reserved

\section{Introduction}

The compactness and efficiency of smartphones puts them at the top of the list of computing devices chosen by users [1-3]. The customization and user-friendliness of Android make this OS so appealing for a large amount of smartphone users. These are the main reasons why MedPlanner is an application developed for smartphones that run the Android operating system. This application runs on a very basic and easy to understand concept. It improves the user's life by reminding them about their medication. Forgetfulness is one of the most common faults that people deal with daily. For example, does one remember with certainty the moment they screwed the toothpaste cap back on or they locked the door. Everybody just assumes they did these things out of habit. These assumptions are, in most cases, not a problem, but what happens when someone skips parts of their 
medical treatment or takes certain medications twice or trice just to be sure? Regardless if the user takes certain medications daily for a very long period of time or just a short one, they can easily forget whether they took them or not. What MedPlanner actually does is that it reminds users about their medication, if they haven't taken it until a specific time of the day. The interface is very simple and can be used with no difficulty even by unexperienced smartphone users. It consists of a main menu and three secondary menus (Morning-, Noon-, Evening-Menu). Each secondary menu has its own list on which the user adds medications and a checkbox that should be checked, if the user took the medication on the list, so that the remind notification will not be sent. The morning notification is sent at $10 \mathrm{o}$ 'clock, the noon one at $16 \mathrm{o}$ 'clock and the evening one at $22 \mathrm{o}$ 'clock. These reminders are sent only if the specific checkbox does not get checked.

The largest part of people that need medications often is also the one that lacks most of the skills required to use more complex applications and software; namely the elderly. The majority of the already existing apps are not easy to work with, if one lacks experience with software or technology in general. This lead to the simplicity of the interface of MedPlanner. Another difficulty could be the language used by applications to give tips. MedPlanner uses images to direct the user and does not require language skills.

\section{User Interface and Application Functionality}

The application starts with a main menu (Fig.1) that has three buttons that lead to the three secondary menus mentioned in the introduction. They can be accessed with a simple tap.

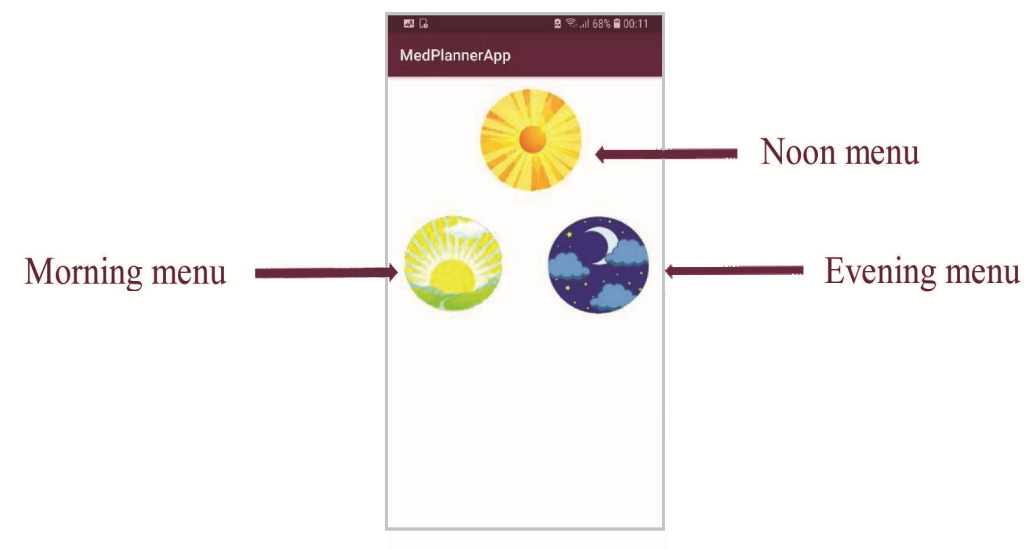

Figure 1. Main menu
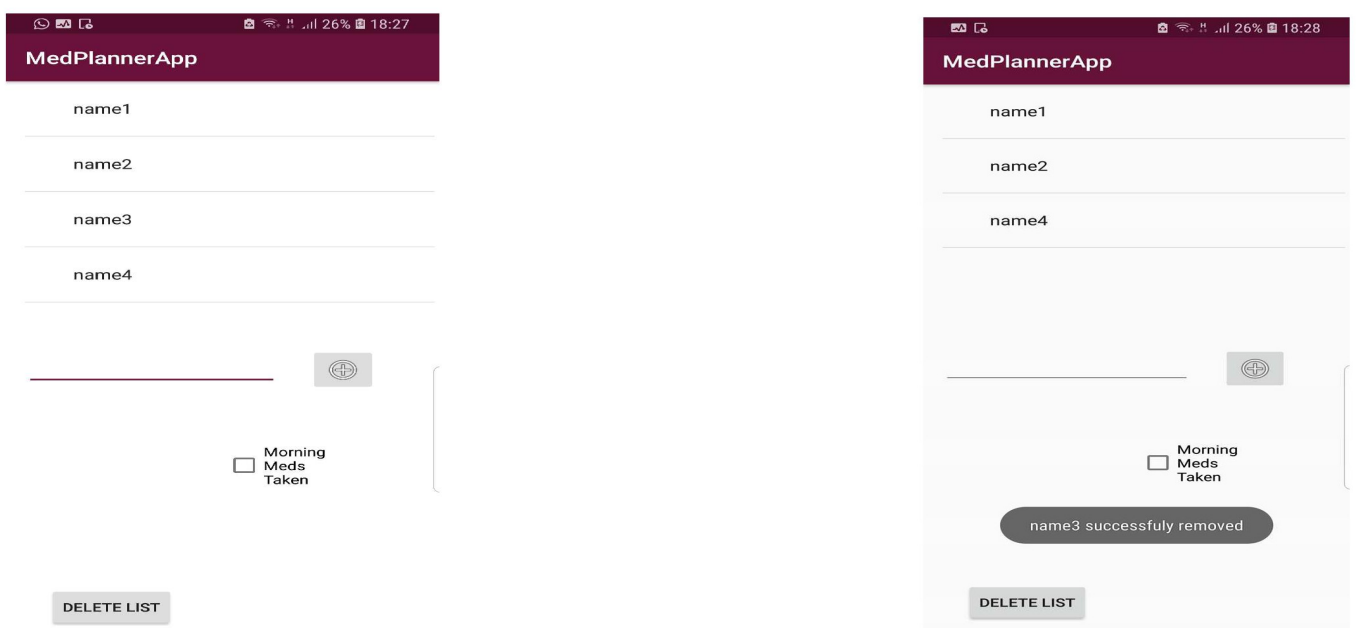

Figure 2. All secondary menus have the same design

Figure 3. Items from the lists can be removed all at once or individually

\begin{tabular}{lllllll}
\hline 34 & Journal of E-Technology & Volume & 9 & Number & 4 & November 2018 \\
\hline
\end{tabular}


After selecting one of the images, the user reaches the respective secondary activity. In the empty field located in the middle of the screen, the users types the medication that gets added to the list after tapping the + button next to the field. The typed name appears in the upper list. If the user took the medications before the specific time limit mentioned before (10,16 and respectively 22 o'clock) they simply check the checkbox located underneath the empty field. The checkboxes for each activity will get unchecked at midnight.

\section{Application Development Software}

\subsection{Programming Environment - Android Studio}

MedPlanner was created with Java-based, Android object-oriented programming language in the Android Studio 3.2.1 environment. It has been tested both on a smartphone and on more virtual devices with the help of Android Emulator.

\subsection{Code Sections}

\subsubsection{Making Medications List}

The application retrieves the name typed by the user, checks if it already exists in the list and if the input field was not null when the user tapped the + button and, if they both conditions are false, it adds the medication to the list.

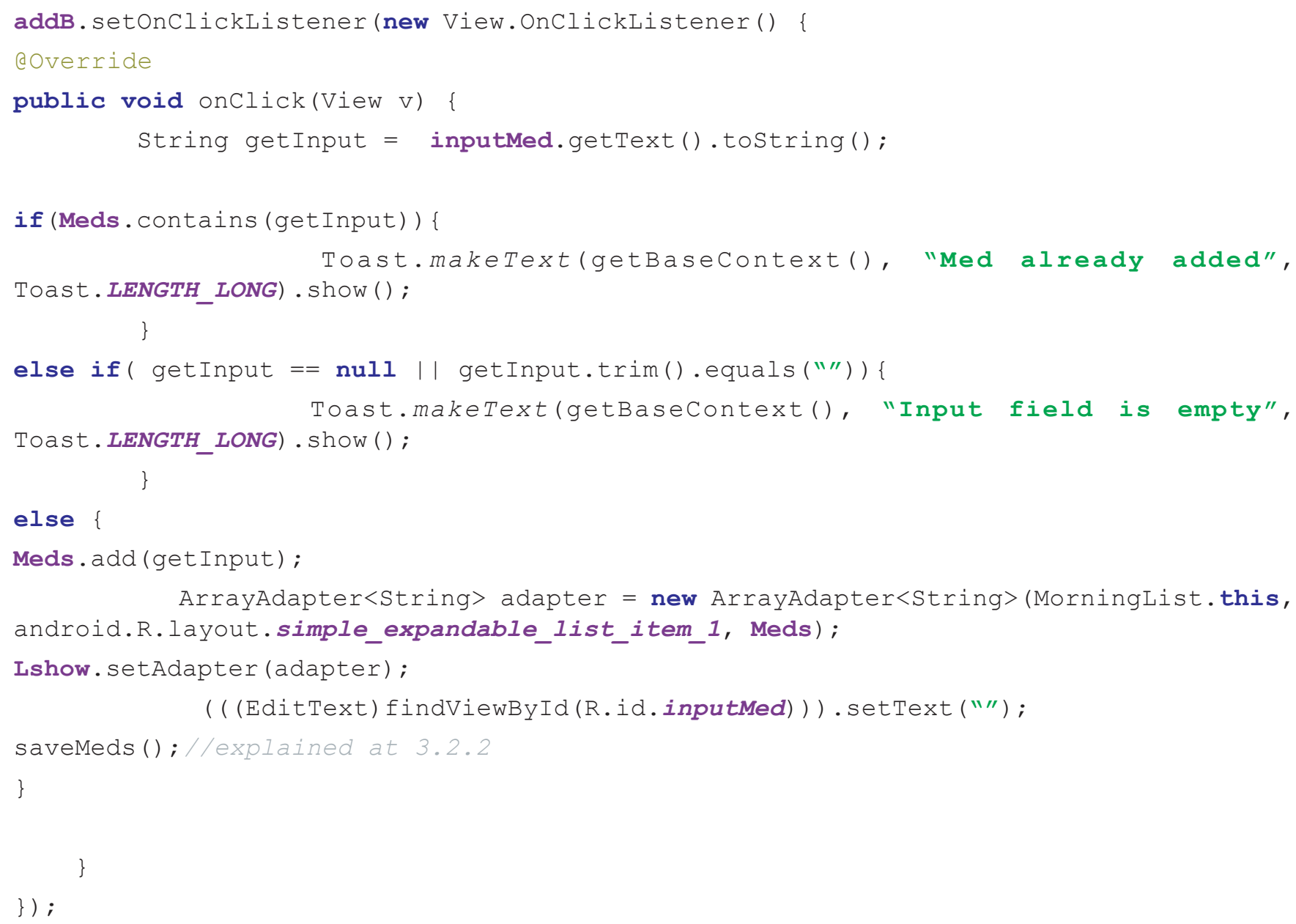

\subsubsection{Saving Medications}

The saveMeds(); method gets called after new medication gets added, in order to save it to a SharedPreferences file. This is necessary because after the app shuts down the array in which the names were saved gets deleted. Every time the app launches the list will be retrieved from the specific file and displayed in a ListView. 


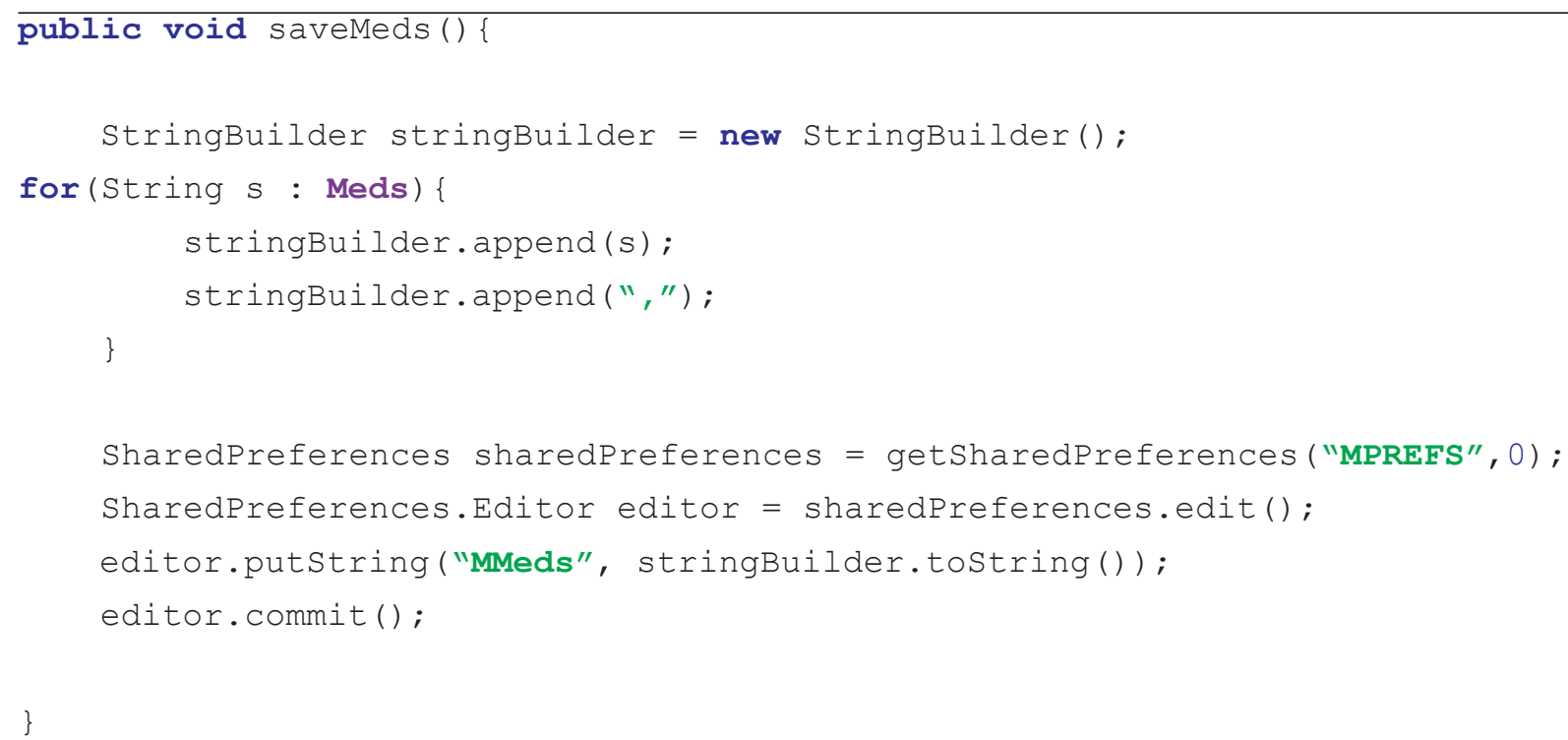

\subsubsection{Alarms}

MedPlanner has 3 alarms and each one launches the remind notification at a specific time. The underlying code section describes the morning notification. The other two alarms are implemented similarly. The application verifies if the current time matches with the set time and if the specific checkbox is checked. A Boolean, that specifies whether the checkbox is checked or not, gets retrieved from a SharedPreference file and depending on its value the alarm gets fired or not[4-7].

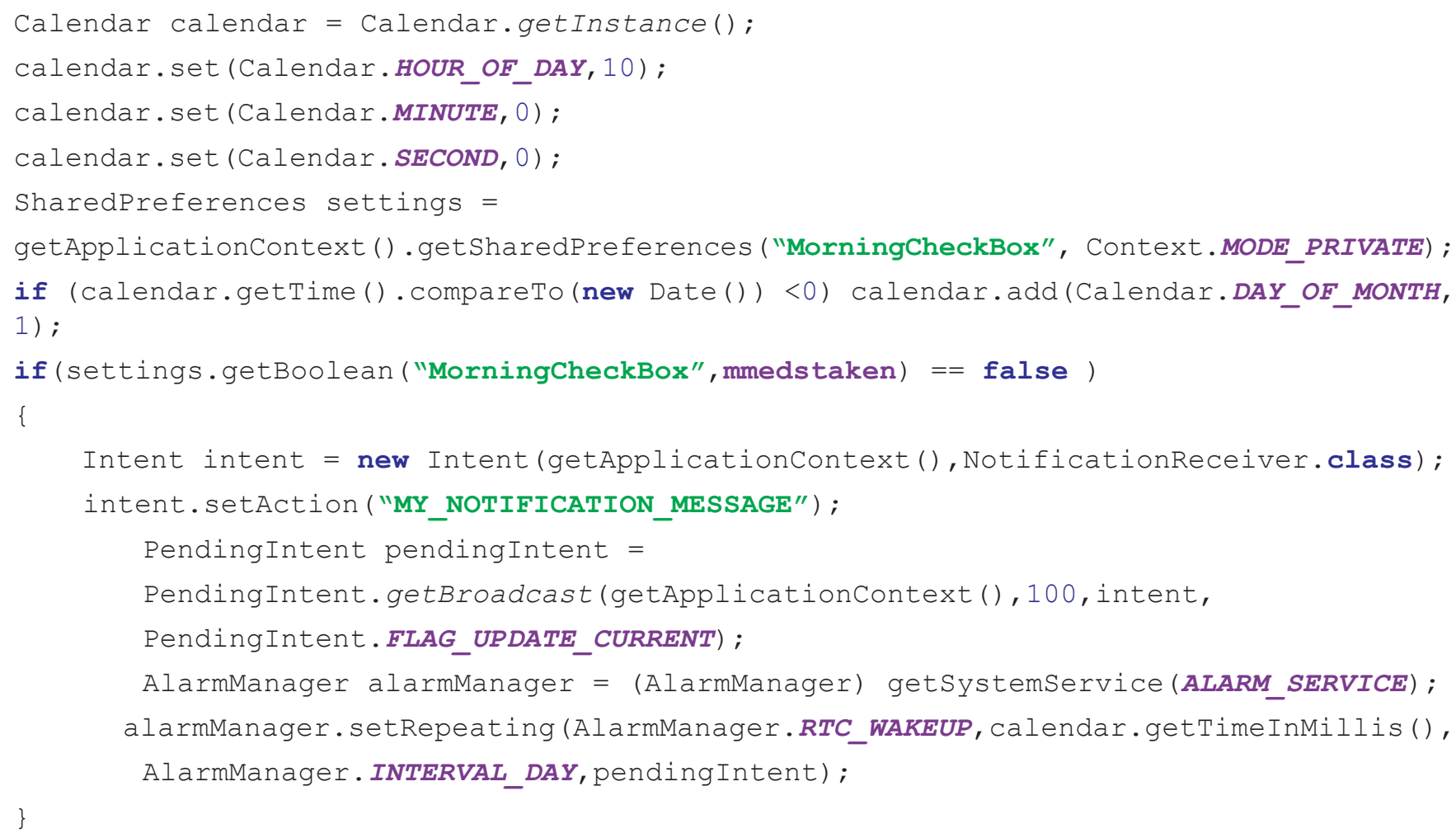

\subsubsection{Sending Notifications}

A Broadcast Receiver receives the alarm and, through it, the Notification Helper class gets reached. Notification Helper is actually 
the one that builds and sends the notification on a channel.

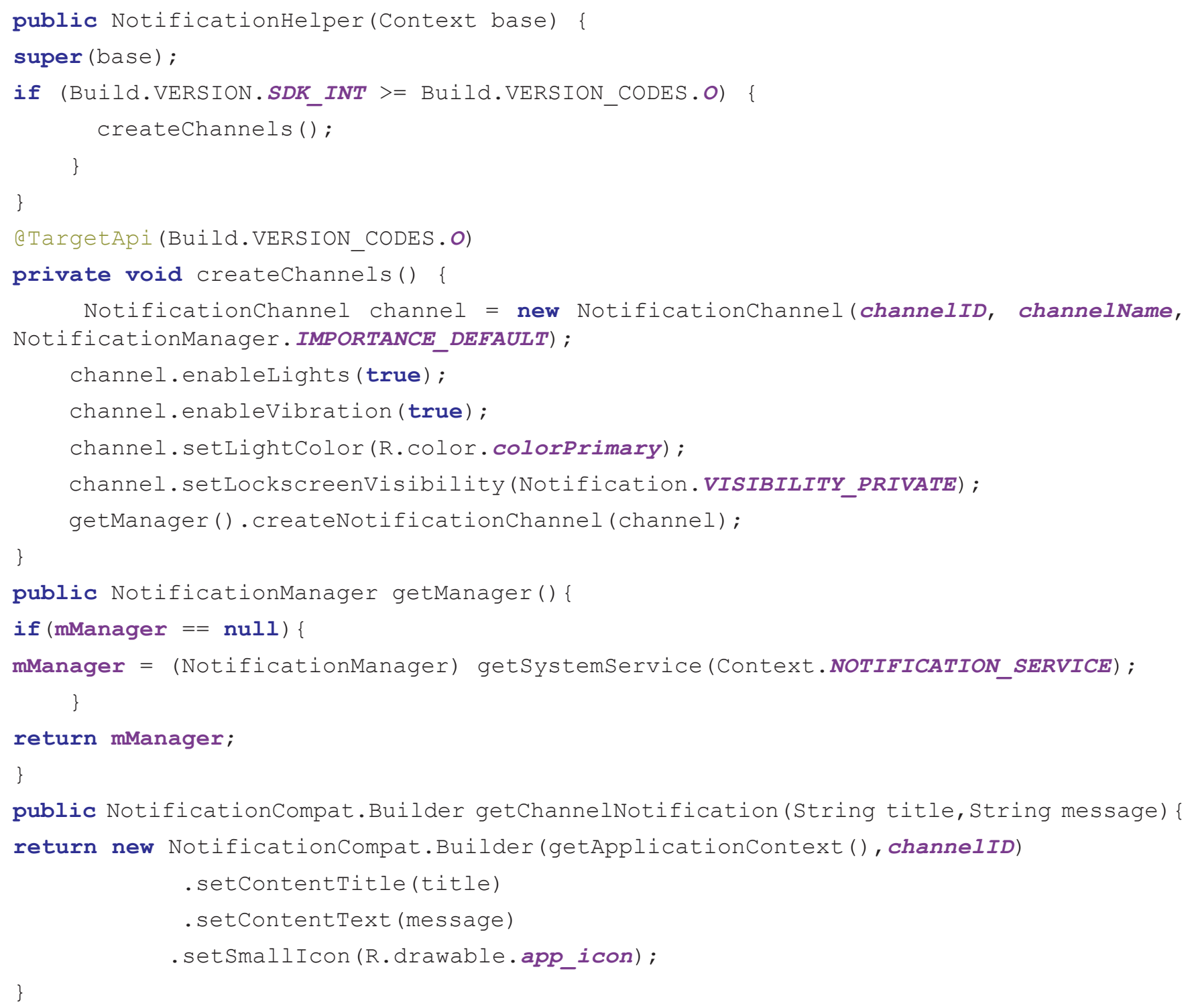

\section{Conclusions and Further Developments}

This paper's purpose is to present MedPlanner, an application that aims to ease users' lives by clearing off a common concern, forgetfulness. This application is suitable for every category of users, does not take up much storage space, does not need permissions that might make users reluctant and has a pleasant and easy to understand design. The notifications appear only if needed and the user does not have to constantly check the application.

MedPlanner is far from its final form and, like everything else, it can be perfected and furtherly developed. Some of the improvements that are planned for the nearby future are adding user accessible settings such as design, a countdown to the end of a prescribed treatment and a database with information about certain medications. All of these will be added while maintaining the simple and user-friendly interface. Furthermore, we plan to test our mobile application on a significant number of elderly persons from Sibiu to see in which proportion it produces the anticipated effects. 


\section{References}

[1] Riley, D. (2012). Using Mobile Phone Programming to Teach Java and Advanced Programming to Computer Scientists, In: Proceedings of the $43^{\text {rd }}$ ACM Technical Symposium on Computer Science Education, p 541-546, North Carolina, USA.

[2] Tigrek, S., Obadat, M. Teaching smartphones programming using (Android Java): Pedagogy and innovation, In: International Conference on Information Technology Based Higher Education and Training (ITHET), p. 1 - 7, 2012, Istanbul, Turkey.

[3] Recode, Two-thirds of adults worldwide will own smartphones next year, 16 October 2017, https://www.recode.net/2017/10/ 16/16482168/two-thirds-of-adults-worldwide-will-own-smartphones-next-year, last accessed $5^{\text {th }}$ February 2019

[4] Stack Overflow, https://stackoverflow.com/questions/45015803/android-o-notification-channels-and-notificationcompat, accessed $14^{\text {th }}$ August 2018

[5] Stack Overflow, https://stackoverflow.com/questions/23024831/android-shared-preferences-example, accessed $22^{\text {nd }}$ July 2018

[6] Android Developer, https://developer.android.com/training/notify-user/channels, accessed $14^{\text {th }}$ August 2018

[7] Android Developer, https://developer.android.com/training/scheduling/alarms, accessed $15^{\text {th }}$ August 2018 